\title{
The newly proposed clinical and post-neoadjuvant treatment staging classifications for gastric adenocarcinoma for the American Joint Committee on Cancer (AJCC) staging
}

\author{
Haejin In $^{1}$ D Ethan Ravetch ${ }^{1} \cdot$ Marisa Langdon-Embry ${ }^{1} \cdot$ Bryan Palis $^{2} \cdot$ Jaffer A. Ajani $^{3} \cdot$ Wayne L. Hofstetter ${ }^{4}$. \\ David P. Kelsen ${ }^{5} \cdot$ Takeshi Sano $^{6}$
}

Received: 3 July 2017 / Accepted: 2 September 2017 / Published online: 25 September 2017

(c) The International Gastric Cancer Association and The Japanese Gastric Cancer Association 2017

\begin{abstract}
Purpose New stage grouping classifications for clinical (cStage) and post-neoadjuvant treatment (ypStage) stage for gastric adenocarcinoma have been proposed for the eighth edition of the AJCC manual. This article summarizes the analysis for these stages.

Methods Gastric adenocarcinoma patients diagnosed in 2004-2009 were identified from the National Cancer Database (NCDB). The cStage cohort included both surgical and nonsurgical cases, and the ypStage cohort included only patients who had chemotherapy or radiation therapy before surgery. Survival differences between the stage groups were determined by the log-rank test and prognostic accuracy was assessed by concordance index. Analysis was performed using SAS 9.4 (SAS, Cary, NC, USA).

Results Five strata for cStage and four strata for ypStage were developed. The 5-year survival rates for cStages were 56.77\%, $47.39 \%, 33.1 \%, 25.9 \%$, and 5.0\% for stages I, IIa, IIb, III, and IV, respectively, and the rates for ypStage were 74.2\%, 46.3\%, $19.2 \%$, and $11.6 \%$ for stages I, II, III, and IV, respectively. The log-rank test showed that survival differences were well stratified and stage groupings were ordered and distinct $(p<0.0001)$. The proposed cStage and ypStage classification was sensitive and specific and had high prognostic accuracy (cStage: $\mathrm{c}$ index $=0.81,95 \%$ CI, 0.79-0.83; ypStage: $\mathrm{c}$ index $=0.80$, 95\% CI, 0.73-0.87).

Conclusion The proposed eighth edition establishes two new staging schemata that provide essential prognostic data for patients before treatment and for patients who have undergone surgery following neoadjuvant therapy. These additions are a significant advance to the AJCC staging manual and will provide critical guidance to clinicians in making informed decisions throughout the treatment course.
\end{abstract}

Keywords Gastric adenocarcinoma $\cdot$ AJCC $\cdot$ Staging $\cdot$ Clinical stage $(c S t a g e) \cdot$ Post-neoadjuvant treatment stage (ypStage)

Haejin In

hin@montefiore.org

1 Department of Surgery, Montefiore Medical Center, Albert Einstein College of Medicine, 1300 Morris Park Ave. Block Building\#112, Bronx, NY 10461, USA

2 NCDB Research Unit, American College of Surgeons, Chicago, IL, USA

3 Department of Gastrointestinal Medical Oncology, The University of Texas MD Anderson Cancer Center, Houston, TX, USA
4 Department of Thoracic and Cardiovascular Surgery, The University of Texas MD Anderson Cancer Center, Houston, TX, USA

5 Department of Medical Oncology, Memorial Sloan Kettering Cancer Center, New York, NY, USA

6 Gastroenterological Center, Cancer Institute Hospital, Tokyo, Japan 


\section{Background}

Gastric adenocarcinoma (GAC) is the fifth most common cancer and the third leading cause of cancer mortality worldwide [1]. In 2012, an estimated 723,000 deaths from GAC occurred globally [1] and 10,730 deaths from GAC occurred in the US [2]. Prognosis and survival vary significantly depending on the stage of disease at the time of diagnosis. Early-stage GAC has a 5-year survival rate of more than $90 \%$, whereas regional GAC confers a 5-year survival of $29 \%$ and metastatic GAC 4\% [3].

The tumor-node-metastasis (TNM) classification system devised by Pierre Denoix in the 1940s was first adopted by the International Union for Cancer Control (UICC) [4]. The American Joint Committee on Cancer (AJCC) Staging and End Results Reporting was organized in 1959. In early editions, the UICC and AJCC proposed different staging systems, but the TNM and stage groupings have been unified since the fourth edition of the UICC and the third edition of the AJCC manual. The rules for classification and staging are updated periodically by the AJCC and UICC and comprise the international standard for staging solid tumors [5].

For the eighth edition of the AJCC Cancer Staging Manual, separate stage grouping classifications for clinical (cStage) and post-neoadjuvant treatment (ypStage) stage were developed on the basis of prognostic information to better provide critical guidance to clinicians in making informed decisions throughout the treatment course. The clinical classification in prior editions was not developed based on survival data but was assigned identically to the pathological TNM classification for purposes of data collection. The assumption that data used to derive pathological classification can be used retrospectively for treatment decision making for patients before the initiation of therapy could be misleading and thus lead to prescribing inappropriate therapies.

There is also a critical need for separate stage groupings and prognostic information for patients who receive neoadjuvant therapy. Increasingly, patients are undergoing neoadjuvant therapy before surgery. Tumor responsiveness to neoadjuvant therapy drives survival outcomes [6,7], and applying prognostic information derived from surgeryfirst patients may not be applicable to these patients.

The eighth edition of the AJCC manual was published in the fall of 2016 and is expected be presented for adoption in late 2017. This edition provides an opportunity to incorporate survival statistics accumulated from years of prospective data collection by the National Cancer Database (NCDB) and refinements to the current pathological staging system made through collaborative work with the International Gastric Cancer Association [8].
The objective of this article is to provide a summary of our analysis that provided the data for the clinical stage groupings (cStage) and post-neoadjuvant therapy stage (ypStage) that were incorporated into the eighth edition of the AJCC manual.

\section{Methods}

\section{Data source}

NCDB data were used to create clinical stage (cStage) and post-neoadjuvant therapy stage (ypStage) groupings. The NCDB is a nationwide oncology database created by the American College of Surgeon (ACoS) and American Cancer Society (ACS) to explore trends in cancer care and to create regional and state benchmarks for quality improvement [9]. The NCDB currently contains data on more than 30 million patients diagnosed between 1985 and 2016 and is considered the largest clinical registry in the world $[9,10]$. The NCDB collects information on patient demographics (age, sex, race, payer, zip code, median income), tumor characteristics (location, size, grade, stage, histology), treatments (receipt of chemotherapy and radiation therapy), and vital status (dead or alive) from 1500 Commission on Cancer (CoC)-accredited hospitals, representing $70 \%$ of newly diagnosed cancer cases in the US. This information is coded according to Facility Oncology Registry Data Standards (FORDS) [11]. Survival is examined as overall survival (OS).

\section{Study population}

The NCDB was used to identify patients with newly diagnosed gastric adenocarcinomas [International Classification of Disease for Oncology, third edition (ICD-O-3): site code, C161-C169; histology codes, 8000-8152, 8154-8231, 8243-8245, 8250-8576, 8940-8950] between 2004 and 2008. Only first or primary cancers were included in the analysis. Analysis was limited to adults of ages 18-79 who received the first course of treatment in the reporting facility (class of case 10-22). This cohort was then used to create two distinct cohorts for the cStage and ypStage analysis. For the cStage cohort, both surgical and nonsurgical cases were included. The ypStage cohort excluded all patients who did not undergo surgery, and of those who did, selected only patients who had received chemotherapy, chemoradiation, or radiation therapy alone before surgery. Cases with incomplete staging information or that could not be converted from the sixth to seventh edition coding schema were excluded from these analyses. 


\section{Analysis}

The GAC subcommittee of the upper gastrointestinal (GI) expert panel had decided a priori that cStage and ypStage would be examined in the broadest groupings that would be useful in a clinical setting and promote the future collection of data, setting the stage for further refinements in stage classifications. Hence, for cStage, cT1a and cT1b were examined as cT1, and cN categories were examined as $\mathrm{cN} 0$ or $\mathrm{cN}+$, where $\mathrm{cN}+$ includes cN1, cN2, and cN3. Kaplan-Meier survival analysis was used to generate survival estimates. Combinations of $\mathrm{cT}$ and $\mathrm{cN}$ were sorted and grouped according to median survival. Any $\mathrm{cT}$ and $\mathrm{cN}$ combination with fewer than 50 patients were considered unstable estimates and assigned to stage group according to the pathological stage schema.

For ypStage, the stage groupings were kept in accordance with the pathological stage to maintain harmonization of the stage classification schema to be used after surgery. Because a limited number of patients were available for analysis, ypStage prognostic information was generated for broad stage groupings of stages I, II, III, and IV.

Survival differences between stage groups were determined by the log-rank test. We assessed discrimination that was representative of the ability of the AJCC seventh and eighth editions staging survival model to differentiate between subjects with and without events. The prognostic accuracy of the proposed cTNM and ypTNM staging systems was assessed using a concordance index. The $C$ statistic for survival analysis is the probability of concordance given usable pairs of which at least one had an event $[12,13]$; it is interpreted as the probability that event group subjects have higher predicted probabilities than nonevent groups. We calculated all usable pairs for a C-index and 95\% confidence limits (CI) using the estimated probabilities of concordance. Analysis was performed using SAS 9.4.

\section{Results}

\section{Patients, tumors, and surgery}

Data for 7306 patients were available for cStage analysis and data of 708 patients were available for ypStage analysis (Appendix 1). Patient demographics are shown in Table 1.

\section{Clinical stage cohort descriptive analysis}

Patients included in cStage analysis had a median age of 64 years (range, 19-79 years) and a slightly greater percentage of males (58\%). Tumors were more often in the pyloric antrum $(24 \%)$, or unspecified $(27 \%)$. Most tumors were classified as cT4a (30\%) or cT4b (29\%) in depth;
Table 1 Clinicopathological features of gastric cancer patients included in the National Cancer Database (NCDB) available for clinical and neoadjuvant staging

\begin{tabular}{|c|c|c|c|c|}
\hline \multirow[t]{2}{*}{ Variable } & \multicolumn{2}{|c|}{$\begin{array}{l}\text { Clini- } \\
\text { cal stage } \\
(n=7306)\end{array}$} & \multicolumn{2}{|c|}{$\begin{array}{l}\text { yp stage } \\
(n=708)\end{array}$} \\
\hline & $n$ & $\%$ & $n$ & $\%$ \\
\hline Median age (years) & 64 & & 60 & \\
\hline \multicolumn{5}{|l|}{ Sex } \\
\hline Male & 4276 & 58.5 & 425 & 60.1 \\
\hline Female & 3026 & 41.4 & 283 & 39.9 \\
\hline Other & 4 & 0.06 & - & - \\
\hline \multicolumn{5}{|l|}{ Site distribution } \\
\hline C16.1: fundus & 431 & 6.0 & 54 & 7.6 \\
\hline C16.2: body of the stomach & 830 & 11.4 & 90 & 12.7 \\
\hline C16.3: pyloric antrum & 1774 & 24.2 & 155 & 22.0 \\
\hline C16.4: pylorus & 246 & 3.4 & 12 & 1.7 \\
\hline C16.5: lesser curvature of the stomach & 780 & 10.6 & 111 & 15.7 \\
\hline C16.6: greater curvature of the stomach & 369 & 5.1 & 28 & 3.9 \\
\hline C16.8: overlapping sites of the stomach & 860 & 11.8 & 112 & 15.8 \\
\hline C16.9: unspecified & 2016 & 27.5 & 146 & 20.6 \\
\hline \multicolumn{5}{|l|}{ Depth of tumor invasion } \\
\hline No tumor & 11 & 0.2 & 25 & 3.3 \\
\hline Mucosa (T1a) & 1644 & 22.5 & 17 & 2.4 \\
\hline Submucosa (T1b) & $-{ }^{\mathrm{a}}$ & $-{ }^{\mathrm{a}}$ & 22 & 3.1 \\
\hline Muscularis propria (T2) & 500 & 6.9 & 110 & 15.6 \\
\hline Subserosa (T3) & 820 & 11.2 & 172 & 24.4 \\
\hline Serosa exposed (T4a) & 2194 & 30.0 & 282 & 39.9 \\
\hline Invasion of adjacent structure (T4b) & 2137 & 29.2 & 80 & 11.3 \\
\hline \multicolumn{5}{|l|}{ Lymph node stage } \\
\hline N0 (0 nodes) & 3512 & 48.0 & 220 & 31.0 \\
\hline N1 (1-2 nodes) & 2673 & 36.5 & 175 & 24.8 \\
\hline N2 (3-6 nodes) & $-{ }^{\mathrm{b}}$ & $-{ }^{\mathrm{b}}$ & 126 & 17.9 \\
\hline N3a (7-15 nodes) & 709 & 9.8 & 125 & 17.6 \\
\hline N3b ( $\geq 16$ nodes $)$ & 412 & 5.7 & 62 & 8.7 \\
\hline \multicolumn{5}{|l|}{ Metastasis } \\
\hline M0 & 4839 & 66.3 & 602 & 85.0 \\
\hline M1 & 2467 & 33.7 & 106 & 15.0 \\
\hline \multicolumn{5}{|l|}{ Resection type } \\
\hline No surgery & 3447 & 47.2 & - & - \\
\hline Total or near-total gastrectomy & 936 & 12.8 & 306 & 43.1 \\
\hline Distal or partial gastrectomy & 1094 & 15.0 & 149 & 21.1 \\
\hline Proximal gastrectomy & 400 & 5.5 & 61 & 8.6 \\
\hline Gastrectomy, unknown type & 1220 & 16.7 & 187 & 26.4 \\
\hline Surgery, not otherwise specified (NOS) & 69 & 0.9 & 3 & 0.4 \\
\hline Local treatment & 128 & 1.8 & 2 & 0.3 \\
\hline Unknown if surgery & 12 & 0.2 & - & - \\
\hline \multicolumn{5}{|l|}{ Systemic therapy before surgery } \\
\hline Chemotherapy only & 290 & 3.8 & 448 & 63.3 \\
\hline Radiation therapy only & 3 & 0.0 & 8 & 1.1 \\
\hline Both chemotherapy and radiation & 94 & 1.2 & 252 & 35.6 \\
\hline No neoadjuvant therapy & 6919 & 95.0 & - & - \\
\hline
\end{tabular}


Table 1 (continued)

\begin{tabular}{|c|c|c|c|c|}
\hline \multirow[t]{2}{*}{ Variable } & \multicolumn{2}{|c|}{$\begin{array}{l}\text { Clini- } \\
\text { cal stage } \\
(n=7306)\end{array}$} & \multicolumn{2}{|c|}{$\begin{array}{l}\text { yp stage } \\
(n=708)\end{array}$} \\
\hline & $n$ & $\%$ & $n$ & $\%$ \\
\hline \multicolumn{5}{|l|}{ Summary of first course of treatment } \\
\hline No treatment & 1359 & 18.6 & - & - \\
\hline Surgery only & 2044 & 27.9 & - & - \\
\hline Surgery + chemo and/or radiation & 2447 & 33.4 & - & - \\
\hline $\begin{array}{l}\text { Chemo- and/or radiation only (no } \\
\text { surgery) }\end{array}$ & 1456 & 20.1 & - & - \\
\hline
\end{tabular}

a Sixth-seventh edition conversion does not allow for T1b designation from sixth edition

${ }^{b} \mathrm{~N} 1$ and $\mathrm{N} 2$ were combined and presented as N2 for purposes of tablulare presentation

$48 \%$ had cN0 disease and $37 \%$ had $\mathrm{cN} 1$ or cN2 disease. $\mathrm{cN} 1$ and $\mathrm{cN} 2$ were analyzed in aggregate because $\mathrm{cN} 1$ and $\mathrm{cN} 2$ could not be distinguished when converting from from the sixth to seventh edition. M1 disease was present in $34 \%$. Of all patients, $53 \%$ received chemotherapy or radiation therapy (or both) as any part of the first course of treatment. Gastrectomy was performed in $51 \%$ of patients; $47 \%$ did not undergo surgery. Of the surgical patients, $5 \%$ received neoadjuvant therapy before gastrectomy.
Post-neoadjuvant therapy stage cohort descriptive analysis

By definition, all patients included in ypStage analysis received preoperative therapy. Most patients (63\%) received only chemotherapy whereas $35 \%$ received both chemotherapy and radiation before surgery. Median age for the whole group was 60 years (range, 18-79 years) and there was a slight male predominance $(60 \%)$. Tumor site distribution is listed in Table 1. Total or near-total gastrectomy was performed on $43 \%$ of patients who underwent neoadjuvant treatment. After neoadjuvant therapy, $41 \%$ of the pathological specimens were ypT4a, 25\% were ypT3, and $16 \%$ were ypT2. Additionally, 25 patients were ypT0, of whom 18 were ypT0N0, 1 was TisN0, and 6 were ypT0N1. There was a fairly even distribution of ypN disease (ypN0, 31\%; ypN1, $25 \%$; ypN2, 18\%; ypN3a, 18\%; ypN3b, 9\%). ypM1 disease was present in $15 \%$.

\section{Clinical stage classification}

\section{T and $\mathbf{N}$ categories and survival}

Median follow-up for clinical stage was 11.8 months. Survival decreased in a stepwise fashion with increasing cT (Fig. 1). The median survival for cT1 was 78.23 months, for cT2 was 77.57 months, for cT3 was 30.23 months, for cT4a was 18.4 months, and for cT4b was 8.41 months. There was an overlap in survival between cT1 and cT2. Median survival
Fig. 1 Cancer-related 5-year and median survival estimates according to $\mathrm{cT}$ classification

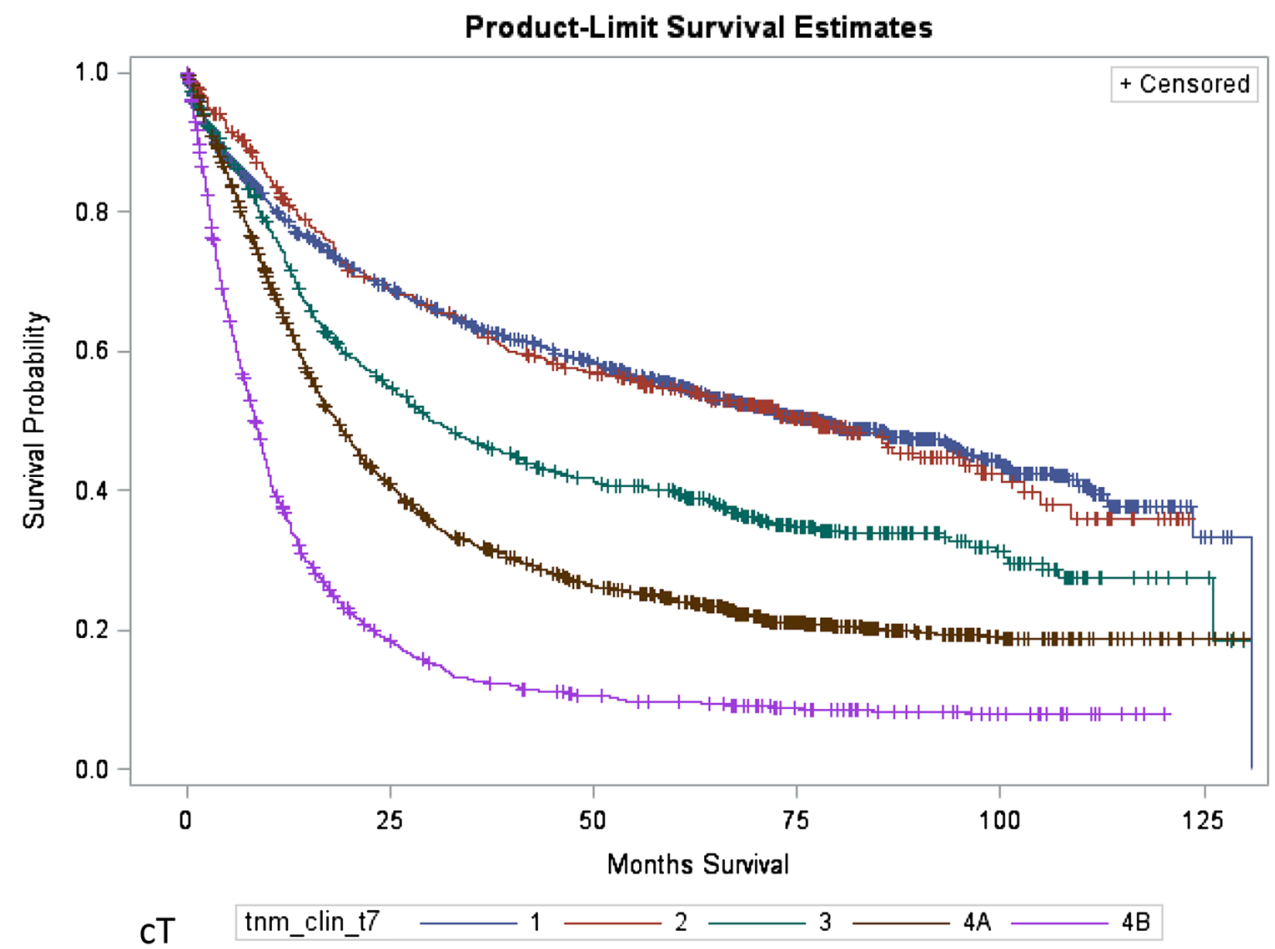


Fig. 2 Cancer-related 5-year and median survival according to $\mathrm{cN}$ classification

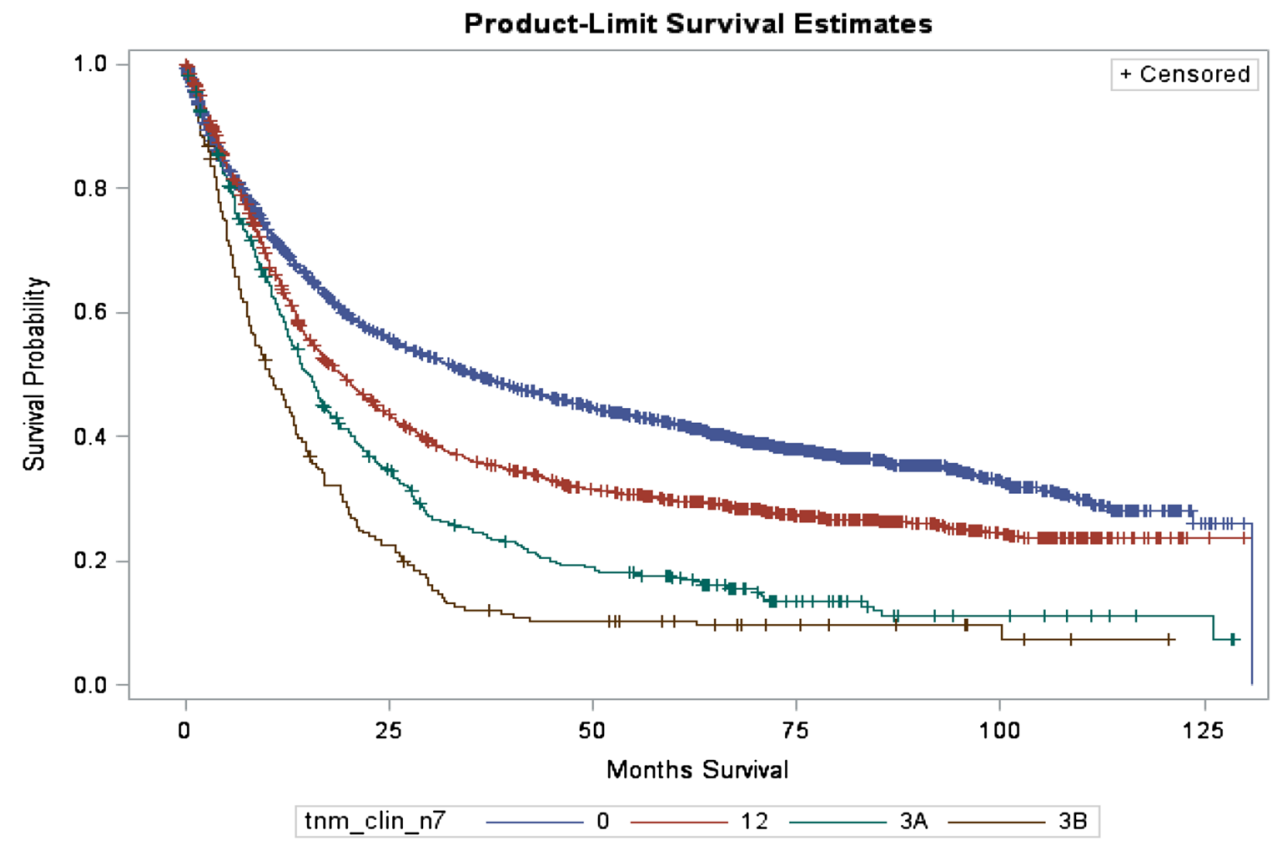

decreased in a similar stepwise fashion with increasing $\mathrm{cN}$. Median survival of patients with cNO (lymph node negative) was 35.25 months, for $\mathrm{cN} 1-\mathrm{cN} 2$ was 19.06 months, for $\mathrm{cN} 3 \mathrm{a}$ was15.21 months, and for $\mathrm{cN} 3 \mathrm{~b}$ was 10.25 months (Fig. 2).

\section{Proposal of cTNM stage grouping}

Patients without evidence of metastatic disease (cM0) were divided into ten groups according to their tumor/ node status by combining the five $\mathrm{T}$ categories (cT1, cT2, cT3, cT4a, cT4b) and the two $\mathrm{N}$ categories (cN0, $\mathrm{cN}+$ ) (Table 2). Patients with metastatic disease (cM1) were analyzed as a separate group. Stage groupings were determined by median survival times. The $\mathrm{cT}$ and $\mathrm{cN}$ combinations were clustered into four stage groups with further subdivision of stage II into IIa and IIb depending on the depth of tumor invasion and extent of lymph node involvement.

cT4bN0 and cT4bN+ were noted to have particularly poor prognosis, nearly that of M1 disease. Hence, T4b was grouped with stage IV. cT1N+ was noted to have a particularly lower survival, 34 months, as compared to cT2N+, 67 months. Although the data would suggest cT1N+ should be grouped with either stage cIIb or cIII, the committee decided to keep the natural progression of stages and retain cT1 + with $\mathrm{cT} 2 \mathrm{~N}+$. This decision was in consideration of keeping this new introduction to the AJCC familiar to users, as well as the data not yet being validated.
Table 2 Five-year survival estimates for proposed cTNM stage classifications

\begin{tabular}{llllllrr}
\hline Clinical stage & $\begin{array}{l}\text { Number of } \\
\text { patients }(n=11, \\
\text { 999) }\end{array}$ & $\begin{array}{l}\text { 1-year } \\
\text { survival } \\
(\%)\end{array}$ & $\begin{array}{l}\text { 3-year } \\
\text { survival } \\
(\%)\end{array}$ & $\begin{array}{l}\text { 5-year } \\
\text { survival } \\
(\%)\end{array}$ & Median survival $^{\mathrm{a}}{ }^{2}$ & $95 \%$ CI & \\
\hline cT1N0M0 & 1,164 & 80.4 & 65.2 & 56.7 & 80.1 & 70.87 & 96.23 \\
cT2N0M0 & 254 & 81.2 & 63.5 & 56.6 & 84.93 & 58.41 & 104.9 \\
cT1N+M0 & 160 & 67.6 & 48.8 & 43.8 & 34.07 & 22.54 & 78.52 \\
cT2N+M0 & 136 & 81.9 & 59.4 & 51.4 & 66.96 & 37.39 & 102.77 \\
cT3N0M0 & 244 & 75.0 & 52.3 & 46.8 & 45.01 & 26.84 & 68.44 \\
cT4AN0M0 & 539 & 65.8 & 36.4 & 26.8 & 20.14 & 17.71 & 24.00 \\
cT3N+M0 & 367 & 72.1 & 42.7 & 34.6 & 28.16 & 21.75 & 33.05 \\
cT4AN+M0 & 1,060 & 64.3 & 29.8 & 22.8 & 17.05 & 15.97 & 19.35 \\
cT4BN0M0 & 396 & 34.6 & 11.2 & 7.5 & 7.72 & 6.34 & 8.84 \\
cT4BN+M0 & 519 & 38.7 & 13.6 & 11.3 & 8.87 & 7.89 & 9.79 \\
cM1 & 2,467 & 25.0 & 5.9 & 3.3 & 5.45 & 5.06 & 5.88 \\
\hline
\end{tabular}

${ }^{\mathrm{a}}$ Months 
The 5-year survival rates for cStage groupings were $56.77 \%$ for stage I, $47.39 \%$ for stage IIa, 33.1\% for stage IIb, $25.9 \%$ for stage III, and $5.0 \%$ for stage IV (Fig. 3). The logrank test showed strong evidence of heterogeneity among the stage groups, indicating that survival differences were well stratified and that true survival differences existed between groups ( $p<0.0001)$. The proposed cTNM staging classification was highly sensitive and specific, and had high prognostic accuracy (concordance index $=0.81 ; 95 \%$ CI, 0.79-0.83).

\section{Post-neoadjuvant therapy stage classification}

Median follow up for ypStage was 22.6 months. The ypStage classification maintained the same classification schema as the proposed AJCC eighth edition pathological stage [14]. The 5-year survival rates for ypStage groupings were $74.2 \%$ for stage I, $46.3 \%$ for stage II, $18.6 \%$ for stage III, and $7.0 \%$ for stage IV (Fig. 4). In addition, there were 18 cases of
Fig. 3 Cancer-related 5-year survival estimates for proposed cTNM stage groups (log-rank test $=2276.83, p<0.0001)$
Fig. 4 Cancer-related 5-year survival estimates for proposed ypTNM stage groups (log-rank test $=180.44, p<0.0001$ )
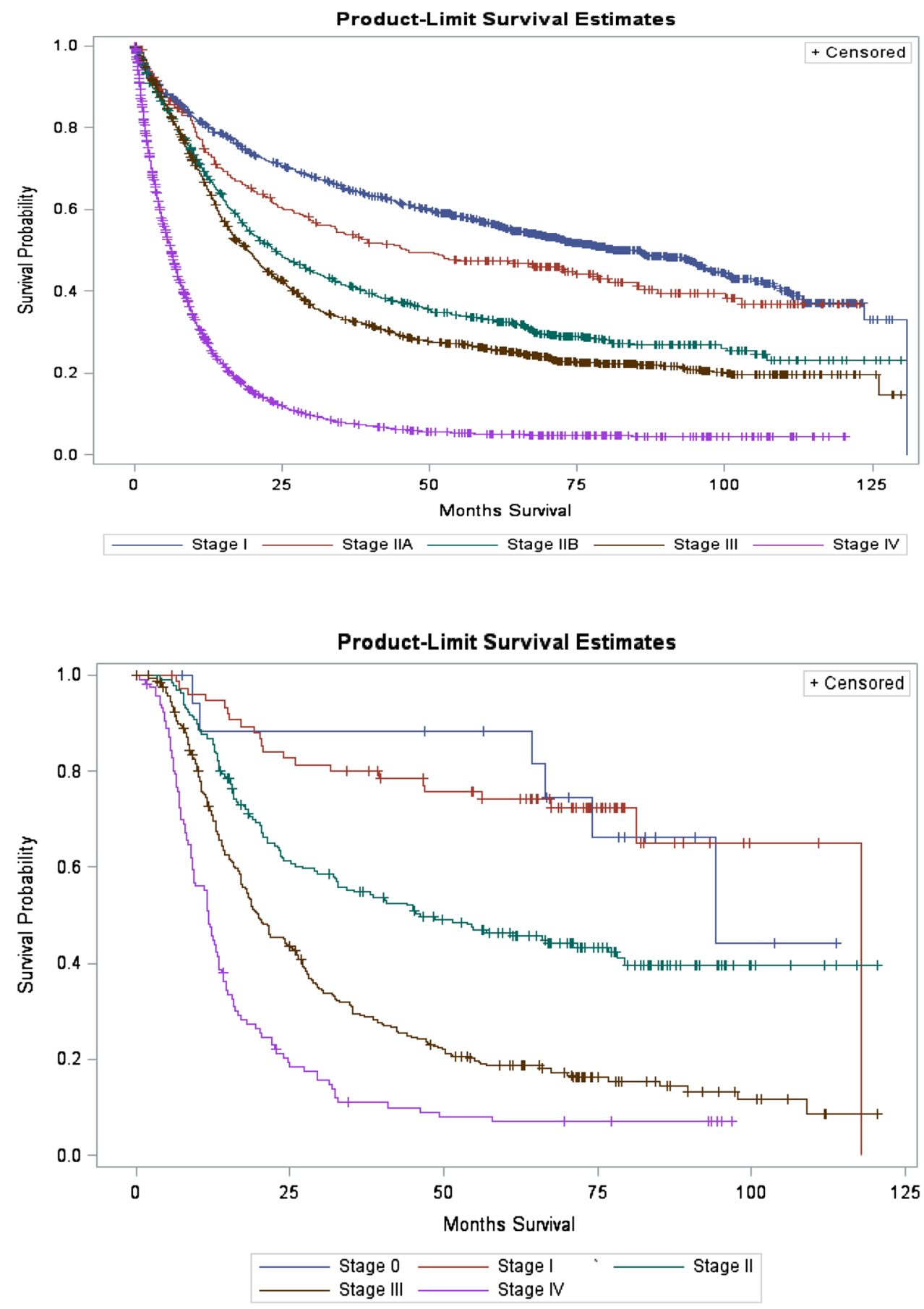
complete response (ypT0N0) and 1 case with ypTisN0. These cases combined had a 5-year survival of $88.8 \%$ (presented as ypStage CR in Fig. 4). The log-rank test showed that survival was well stratified among the stage groups $(p<0.0001)$. The proposed ypTNM staging system had high prognostic accuracy (concordance index $=0.80 ; 95 \%$ CI, 0.73-0.87). Figure 5 presents the newly proposed cTNM and ypTNM stage classifications.

\section{Discussion}

The utility of a cancer staging schema is to associate a disease with prognosis and therapy, thereby providing clinicians with relevant patient data with which to make decisions. The proposed eighth edition of the AJCC staging manual compartmentalizes GAC into three platforms from which we approach the disease: the clinical stage (cStage) to be used to provide prognostic information and inform treatment decisions after staging workup has been completed, the pathological stage (pStage) to provide prognostic risk assessment using tumor specimen analysis and inform adjuvant treatment decisions, and the post-neoadjuvant therapy stage (ypStage) to provide a prognostic risk assessment for tumors that have been subjected to neoadjuvant therapy.

In prior versions, only pathological stage was provided for GAC staging. Pathological stage derives information

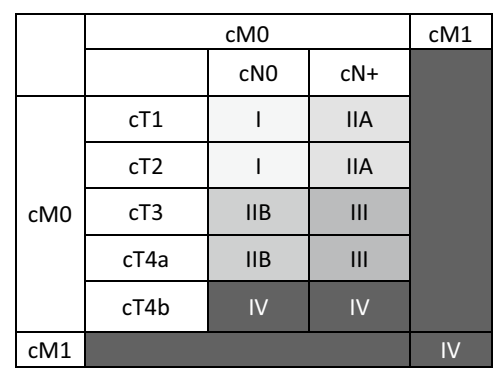

\begin{tabular}{|c|c|c|c|c|c|}
\hline \multicolumn{5}{|c|}{ ypMo } & \multirow[t]{2}{*}{ ypM1 } \\
\hline & $\begin{array}{c}\text { ypNo } \\
(0)\end{array}$ & $\begin{array}{l}\text { ypN1 } \\
(1-2) \\
\end{array}$ & $\begin{array}{l}\text { ypN2 } \\
(3-6)\end{array}$ & $\begin{array}{c}\text { ypN3 } \\
(>6)\end{array}$ & \\
\hline урT1 & 1 & 1 & II & II & \\
\hline урT2 & 1 & II & II & III & \\
\hline урT3 & II & II & III & III & \\
\hline урT4а & II & III & III & III & \\
\hline урT4b & III & III & III & III & \\
\hline
\end{tabular}

Fig. 5 Proposed cTNM and ypTNM stage classification exclusively from surgically resected cases of GAC to provide prognostic information for gastric cancer. In the absence of clinical staging information, treating physicians have used pathological stage data to clinically stage and devise treatment strategies. However, the inherent inaccuracies in clinical staging modalities can cause a fundamental discordance between outcomes based on clinical staging compared to that from pathological stage. Furthermore, the data presented in the pathological stage carry an implicit patient selection bias. Because patients who have undergone surgery are generally younger and healthier, the use of survival statistics based on these patients for treatment decisions after staging workup (before treatment) has led to overly optimistic predictions. Clinical stage for the eighth edition was created using the NCDB, which captures $70 \%$ of all cancer cases diagnosed in the US; $47 \%$ of patients in the dataset used for the creation of clinical stage did not undergo surgery, providing real-world prognostic information that can be used by patients and clinicians to better inform management decisions. As a case in point, in the proposed eighth edition, the projected 5-year survival for pStage IA and IIA is $94 \%$ and $88 \%$, respectively, whereas cStage I based on the NCBD was much lower, at 56\%. Similarly, pStage IIB was $68 \%$ whereas cStage IIB was only 33\% [14]. There are several potential reasons for these survival disparities, including selection bias, information gaps, and inaccuracies in staging modalities.

The AJCC eighth edition also provides an alternative clinical stage prognostic table derived from surgically treated patients treated at Shizouoka Cancer Center (Japan) $[14,15]$. The use of two data sources provided validation for our clinical stage grouping schema with excellent separation of stage groups from both sources while highlighting differences in prognosis based on clinical stage derived from all patients or only surgical patients and by differences in the patient population (US versus Japanese patients).

As neoadjuvant therapy is becoming increasingly common and has been demonstrated to be effective when combined with adjuvant therapy in localized GAC [16], there is a need for revising the AJCC staging. The development of a post-neoadjuvant stage was presaged by a number of prior studies. Data compiled from two prospective trials of preoperative chemotherapy and radiation therapy $[17,18]$ showed that when patients with localized GAC received neoadjuvant therapy, the degree of pathological response and not the stage preceding treatment dictated patient outcome [18]. Patients achieving pathological complete response (pathCR) 
or partial response (pathPR) had a significantly longer median survival time (63.9 months) than those achieving less than pathPR (12.6 months; $p=0.03$ ) [7]. Cancers having responded to neoadjuvant therapy can be downstaged from the baseline clinical stage, suggesting a cancer biology that is more responsive to chemotherapy. On the other hand, cancers that are less responsive after neoadjuvant therapy demonstrate therapeutic resistance with associated aggressive metastatic and invasive potential. The ypStage proposal in the AJCC eighth edition allows for a modification of their prognosis based on tumor responsiveness to therapy, providing a critical insight into the future management and outlook for these patients.

Given the lack of ypStage in staging manuals for gastric cancer, prognostic guidance on patients with complete response to neoadjuvant treatment had been lacking. Our current analysis includes 19 patients with complete response of their primary tumor (ypT0N0 or ypTisN0). Although the small number of cases in this category cannot produce stable estimates, it shows a trend toward favorable outcomes of 5 -year survival of $89 \%$. The notation of these complete responders within the staging schema is currently under debate. As described in the chapter 'Principles of Cancer Staging' of the AJCC manual [14], stage 0 is used to indicate carcinoma in situ (pTisN0M0), and no stage is assigned if the specimen shows no cancer. However, the lack of cancer in the resected specimen is very different in the context of a tumor having received treatment compared to an untreated specimen. The former situation would indicate that the cancer is highly responsive to treatment, but the latter would indicate a misdiagnosis. UICC considers ypTONOM0 as stage 0 [19], and, given the need for harmonization of AJCC and UICC, denoting ypTONOM0 as stage 0 is under consideration. For purposes of this article, ypT0N0M0 and ypTisN0M0 are combined in tables and figures and presented as ypStage CR.

The data used to create cStage and ypStage were from NCDB and have the limitations contained in the shortcomings of this database. Namely, the data for NCDB are collected by cancer registrars for the purpose of maintaining a cancer registry but not specifically for research purposes. In addition, these new staging recommendations were developed with data obtained from a nonstandardized approach to diagnosis, staging, and surgery, with particular variability regarding nodal resection [20]. GAC is approached differently depending on the cancer center, the resources available, and the expertise of the clinician [21]. At the same time, this is also one of the strengths of this analysis in providing data for the clinical stage because it contains the same staging methodologies and approaches currently used in practice across the US. Also, for this inaugural proposal of cStage and ypStage, the data were not sufficiently robust to provide more granular substages. In addition, patients who were not fully coded (e.g., Tx, $\mathrm{Nx}$ ) and those that could not be converted from the sixth to seventh edition were excluded, resulting in the loss of information provided by these patients. In particular, cT and $\mathrm{cN}$ were often incomplete, with the result that only $30 \%$ of cases $(8,001$ of 26,516$)$ were available for clinical stage analysis. Patients without complete staging information may represent patients who had an incomplete workup before surgery, either because they were not surgical candidates or needed emergency surgery, or this discrepancy may represent systematic issues such as some institutions routinely underreporting clinical stage. As coding requirements become more stringent and consecutive editions of the AJCC manual take into account conversion issues between cancer staging editions, we anticipate less information loss and greater ability to develop granular stratification of the staging data in subsequent editions.

\section{Conclusions}

In summary, the proposed eighth edition establishes two new staging schemata that provide essential prognostic data for patients before treatment and for patients who have undergone surgery following neoadjuvant therapy. The recognition of a vast population previously uncaptured by a solely pathological approach to gastric cancer will improve the information provided for our patients and aid in treatment decisions. Future progress in our understanding of GAC pathogenesis will be aided by these additions, and the attention brought by the addition of a cStage and ypStage to the AJCC staging manual will undoubtedly improve the accuracy of pretreatment staging for GAC patients as well as providing more accurate prognostic information for patients given neoadjuvant therapy and should be considered a significant advance.

\section{Compliance with ethical standards}

Conflict of interest The authors declare that they have no conflict of interest.

Ethical standards This article does not contain any studies with human or animal subjects performed by any of the authors. 


\section{Appendix 1. Selection criteria for patient data extracted from the National Cancer Database (NCDB)}

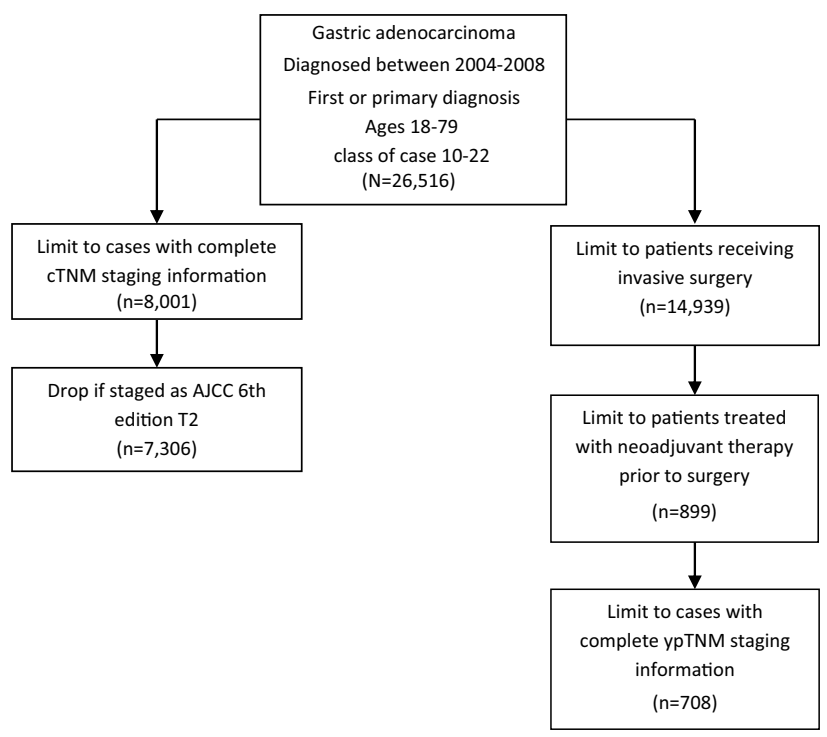

\section{References}

1. World Health Organization. Cancer fact sheet number 297. 2015. Available from: http://www.who.int/mediacentre/factsheets/fs297/ en/. Accessed 10 Mar 2016.

2. American Cancer Society. What are the key statistics about stomach cancer? 2016. Available from: http://www.cancer.org/cancer/stomachcancer/detailedguide/stomach-cancer-key-statistics. Accessed 4 Dec 2016.

3. Ries L, Harkins D, Krapcho M, Mariotto, A, Miller BA, Feuer EJ et al. (2006) SEER cancer statistics review, 1975-2003. Available from: http://seer.cancer.gov/csr/1975_2003/. November 2005 SEER data submission.

4. Kennedy BJ. T N M classification for stomach cancer. Cancer 1970;26(5):971-83. doi:10.1002/10970142(197011)26:5<971::AID-CNCR2820260503>3.0.CO;2-R.

5. American Joint Committee. Manual for staging of cancer. 1st edn. Chicago: American Joint Committee for Cancer Staging and End Results Reporting; 1977.

6. Ajani JA, et al. Preoperative and postoperative combination chemotherapy for potentially resectable gastric-carcinoma. J Natl Cancer Inst. 1993;85(22):1839-44.
7. Ajani JA, et al. Multi-institutional trial of preoperative chemoradiotherapy in patients with potentially resectable gastric carcinoma. J Clin Oncol. 2004;22(14):2774-80.

8. Sano T, Aiko T. New Japanese classifications and treatment guidelines for gastric cancer: revision concepts and major revised points. Gastric Cancer. 2011;14(2):97-100.

9. National Cancer Data Base. Available from: https://www.facs.org/ quality\%20programs/cancer/ncdb. 20 June 2017.

10. Bilimoria KY, Stewart AK, Winchester DP, Clifford KY. The National Cancer Center Database: a powerful initiative to improve cancer care in the United States. Ann Surg Oncol. 2008;15(3):683-90.

11. Commission on Cancer of the American College of Surgeons. Facility oncology registry data standards revised for 2016. Chicago, IL: American College of Surgeons; 2016.

12. Pencina MJ, D'Agostino RB. Overall C as a measure of discrimination in survival analysis: model specific population value and confidence interval estimation. Stat Med. 2004;23:2109-23.

13. Liu L, Forman S, Barton B (2009) Fitting Cox model using PROC PHREG and beyond in SAS. SAS Global Forum. March 22-25 2009; Washington, DC.

14. American Joint Committee on Cancer. AJCC Cancer Staging Manual. 8th ed. Heidelberg: Springer; 2017. p. 1024.

15. Bando E, Makuuchi R, Tokunaga M, Tanizawa Y, Kawamura T, Terashima M (2017) Impact of clinical tumor-node-metastasis staging on survival in gastric carcinoma patients receiving surgery. Gastric Cancer 20(3):448-456. doi:10.1007/s10120-016-0637-x.

16. Li W, et al. Neoadjuvant chemotherapy for advanced gastric cancer: a meta-analysis. World J Gastroenterol. 2010;16(44):5621-8.

17. Ajani JA, et al. Paclitaxel-based chemoradiotherapy in localized gastric carcinoma: degree of pathologic response and not clinical parameters dictated patient outcome. J Clin Oncol. 2005;23(6):1237-44.

18. Rohatgi PR, et al. Surgical pathology stage by American Joint Commission on Cancer criteria predicts patient survival after preoperative chemoradiation for localized gastric carcinoma. Cancer (Phila). 2006;107(7):1475-82.

19. Wittekind C, Compton C, Brierley J, Sobin LH. TNM Supplement. 4th edn. Hoboken NJ: Wiley-Blackwell; 2012.

20. Greenberg CC, Lipsitz SR, Neville B, In H, Hevelone N, Porter SA, et al. Receipt of appropriate surgical care for Medicare beneficiaries with cancer. Arch Surg. 2011;146(10):1128-34. doi:10.1001/archsurg.2011.141.

21. Cravo M, Fidalgo C, Garrido R, Rodrigues T, Luz G, Palmela C, et al. Towards curative therapy in gastric cancer: faraway, so close! World J Gastroenterol. 2015;21(41):11609-20. doi:10.3748/wjg. v21.i41.11609. 